\title{
Self Heating of an Atomic Force Microscope
}

O. Kučera

Atomic force microscopy (AFM) is a sensitive technique susceptible to unwanted influences, such as thermal noise, vibrational noise, etc. Although, tools that protect AFM against external noise have been developed and are widely used, there are still many sources of inherent noise. One of them is self-heating of the apparatus. This paper deals with self-heating of the AFM using an optical lever. This phenomenon is shown to be substantial in particular after activation of the microscope. The influence on the intrinsic contact noise of AFM's is also examined.

Keywords: Atomic force microscopy, noise.

\section{Introduction}

Atomic force microscopy (AFM) [1, 2] senses the interaction forces between a sharp probing tip (with a radius of curvature from about ones to tens of nanometres) and the surface of the sample. The principle of AFM is shown in the Fig. 1.

The probing tip is attached to the free end of a cantilever type spring (about tens or hundreds of micrometres in length). The forces between the probing tip and the sample (of an order of magnitude of $10^{-13}$ to $10^{-4} \mathrm{~N}$ ) deflect the cantilever, which is monitored while the sample is being moved under the tip. The topography of the sample is subsequently reconstructed on the basis of the deflection signal that is recorded. Another reconstruction method applies a signal with its origin in the feedback loop which is used to keep the deflection of the cantilever constant. AFM is also widely used as a non-imaging technique [3] enabling measurements of the local mechanical properties of a sample, such as Young's modulus, etc. AFM supports 3 modes of operation, which can be derived from the force-distance curve of the sample-tip couple (see Fig. 2). The contact regime (sometimes referred to as $\mathrm{C}-\mathrm{AFM}$ ), which is referred to in this paper, operates in the range of the repulsive forces. In the false engage mode, which is neither an imaging regime nor a force measurement regime, the cantilever is kept unloaded in the air.

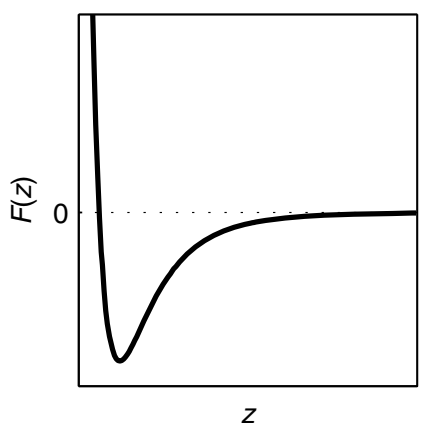

Fig. 2: Force-distance curve (arbitrary units) with the typical shape of the Lennard-Jones two-body potential

Most AFM systems use an optical lever (sometimes referred to as laser beam deflection) to detect the deflection of the cantilever $[4,5]$ (see Fig. 1). The laser beam from a laser diode is reflected from the free end of the cantilever to the po-

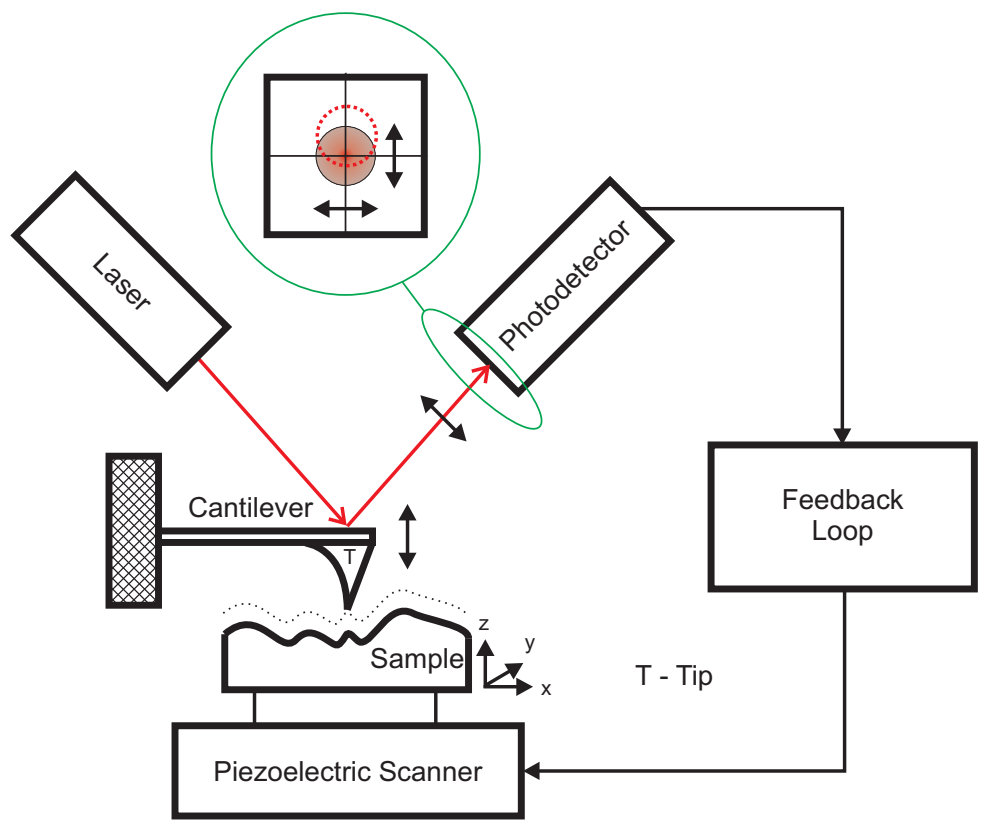

Fig. 1: The principle of AFM 
sition sensitive photo detector (PSPD). The PSPD consists mostly of four photodiodes forming a quad (see the encircled detail in Fig. 1). The difference of the corresponding voltages (induced by the reflected laser beam) on the diodes represents signals used to calculate the deflection and torsion of the cantilever. These signals are termed vertical (deflection) and horizontal (torsion) voltages.

Since an AFM is capable of imaging the sample topography on an atomic scale, or of sensing forces as weak as $10^{-13} \mathrm{~N}$, the issue of noise influencing the resolution and the sensitivity of an AFM has become vitally important [6]. On the onehand, external noise such as acoustic and electromagnetic noise, fluctuations of temperature, vibrations of the building in which the microscope is housed, etc. have an effect on the apparatus. On the other hand, AFM itself generates endogenous noise form several sources. This includes the effects of the electronics, self heating and thermal expansiveness of the AFM components, inherent thermally induced oscillations of the cantilever, etc. which influence the resolution and the sensitivity as well.

This study demonstrates how the PSPD signal evolves versus time after activation of the system, and how the intrinsic contact noise of the AFM's is affected.

\section{Materials and methods}

A Veeco MultiMode IV scanning probe microscope with a Veeco NP20 D cantilever was used in the false-engage and contact regime. The microscope was housed in a special temperature-stabilised room $\left(24^{\circ} \mathrm{C}\right)$ on the basement floor and placed on a vibration isolated table. Temperature stabilisation was provided by a standard air-conditioning unit (AC). The AC had been running for more than one day at the beginning of the experiment, so the room was assumed to be temperature stabilised.

The PSPD vertical and horizontal voltages were recorded in the false-engage mode for 200 minutes after activation of the AFM. To reveal the effect of air-conditioning on these voltages, the AC was turned off from time $t=120 \mathrm{~min}$ till $t=180 \mathrm{~min}$.

The intrinsic contact noise was measured as follows [6]. The probing tip was brought into contact with the sample (a metal washer) and the primary feedback loop was turned off.
The intrinsic contact noise signal was observed by recording the deflection of the cantilever (the PSPD vertical voltage) with the scan area set to $0 \mathrm{~nm}$. This means that the tip was not moving laterally across the surface of the sample. The observed signal was sampled with sampling frequency of $60.621 \mathrm{kHz}$ for $8.6486 \mathrm{~s}$.

\section{Results and discussion}

As shown in Fig. 3, both the horizontal and the vertical voltages begin to decrease directly after activation of the AFM at time $t=0$. The exponential decrease of the voltage vanished at approx. $t=30 \mathrm{~min}$. Until the air-conditioning was turned off at time $t=120 \mathrm{~min}$ both voltages occurred in a stationary state and oscillated slightly. The reason for these oscillations is not clear, but it probably springs from imperceptible temperature changes caused by the air-condition cycle. At $t=120 \mathrm{~min}$, as mentioned above, the AC was turned off. Both voltages started to decrease at the same time. The decrease was reversed by restarting of the AC at $t=185 \mathrm{~min}$. This proves the effect of temperature changes on the vertical and horizontal voltage of the PSPD. Since the temperature of the environment was not changing significantly at the beginning of the experiment (up to $t=120 \mathrm{~min}$ ), the exponential downward phase of the PSPD voltage in this period must be due to self-heating of the AFM. Two explanations for this phenomenon are suggested. Firstly, the laser beam could have heated the PSPD. Secondly, the laser beam could have heated the cantilever, which thus changed its geometry and deformability.

The intrinsic contact noise (ICN) that was observed includes three types of components. Firstly, pure contact noise with small amplitude of ones of the resolution quantum of the $\mathrm{A} / \mathrm{D}$ converter of the AFM. This noise contains the electrical noise of the PSPD, the electromechanical noise of the piezoscanner, and the thermally induced mechanical noise of the sample-tip-cantilever system. Secondly, external noise is also included. This noise is expressed as fluctuations of the general drift, which is the third component.

As noted above, the correlation between PSPD voltage and AC status proved that there is a thermal cause for the PSPD voltage fluctuations. The same temperature changes are the most probable source of the general drift of the ICN;

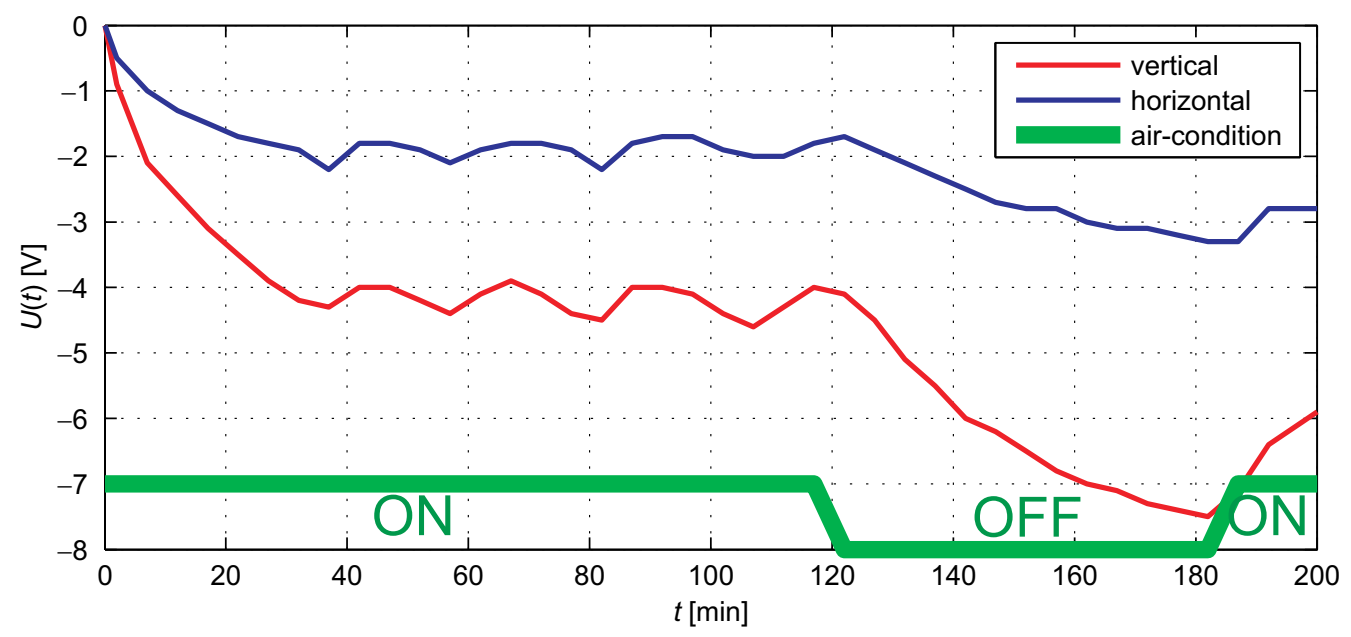

Fig. 3: Influence of self-heating of AFM on the voltage of the deflection detector versus time 


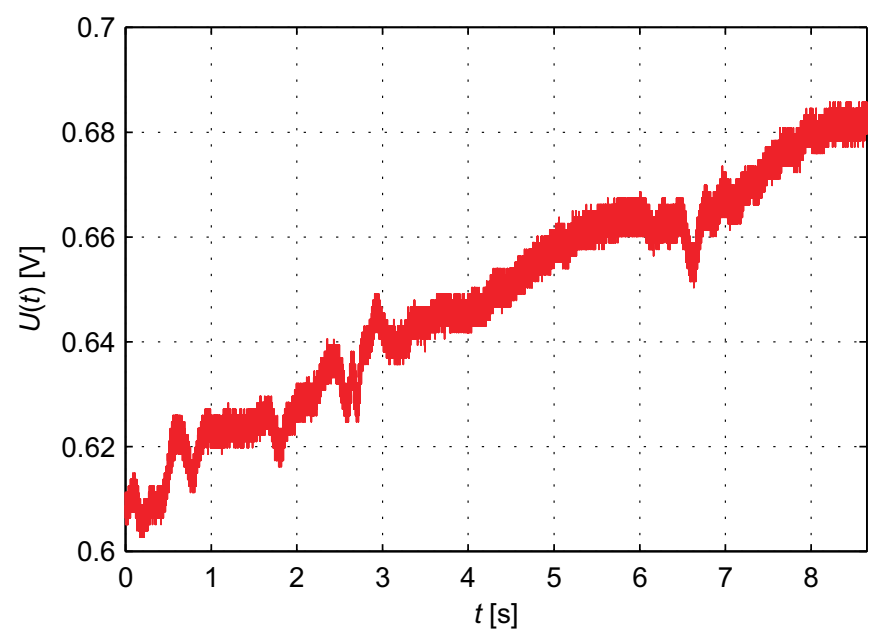

Fig. 4: An example of pure contact noise superimposed on the drift of PSPD voltage. Sudden fluctuations are caused by external noise such as acoustic noise, etc.

however, the mechanism could be somewhat different, because the effect of using the piezoscanner must be taken into account. Note that the differences of the mean voltages in both cases (Fig. 3 and Fig. 4 respectively) are caused by the different AFM regime that is used and the bias voltage applied in the C-AFM. We may thus conclude that the pure contact noise is superimposed on a drift of the PSPD signal, which seems likely to be caused by temperature changes. This feature of ICN measurements, and of AFM measurements generally, is not widely known and it may lead to incorrect determination of noise characteristics such RMS value of the noise, etc. It can also have a negative influence on the vertical resolution of AFM, because PSPD voltage is used to calculate the topography of the sample. In particular, precise force measurements can be totally devalued due to self-heating of the AFM.

\section{Conclusion}

A major problem of AFM measurements (especially on an atomic scale) is the influence of any kind of noise. This study has shown the noise characteristics of AFM, such as RMS and ICN, are strongly influenced by self-heating when an optical lever is used.

\section{References}

[1] Binnig, G., Quate, C., Gerber, Ch.: Atomic Force microscope. Physical Review Letters, Vol. 56 (1986), p. 930-933.

[2] Meyer, E., Heinzelmann, H.: Scanning Force Microscopy. In: Scanning Tunneling Microscopy II (Editors: Weisendanger, R., Güntherodt, H.-J.). Berlin - Heidelberg - New York: Springer, 1992, 1995 (second edition).

[3] Butt, H., Cappella, B., Kappl, M.: Force Measurements with the Atomic Force Microscope: Technique, Interpre- tation and Applications. Surface Science Reports, Vol. 59 (2005), No. 1-6, p. 1-152.

[4] Meyer, G., Amer, N.: Novel Optical Approach to Atomic Force Microscopy. Applied Physics Letters, Vol. 53 (1988), p. 1045-1047.

[5] Alexander, S. et al.: An Atomic-Resolution Atomic-Force Microscope Implemented Using an Optical Lever. Journal of Applied Physics, Vol. 65 (1989), p. 164-167.

[6] Han, W., Lindsay, S.: Intrinsic Contact Noise: A Figure of Merit for Identifying High Resolution AFMs. Applicatin Note, Agilent Technologies, 2007.

[7] Wilkening, G., Koenders, L.: Nanoscale Calibration Standards and Methods. Weinheim: WILEY-VCH Verlag GmbH \& Co. KGaA, 2005.

[8] Kučera, O.: Oscillation States on Yeast Cell Membranes. Diploma thesis, Praha: Czech Technical University in Prague, 2008.

Ing. Ondřej Kučera

phone: +420224352820

e-mail: kucerao@ufe.cz

Department of Circuit Theory

Czech Technical University in Prague

Faculty of Electrical Engineering

Technická 2

16627 Prague 6, Czech Republic

Institute of Photonics and Electronics Academy of Sciences of the Czech Republic

Chaberská 57

18251 Prague 8, Czech Republic 